\title{
Respiratory physiotherapy in the bronchiectasis guidelines: is there a loud voice we are yet to hear?
}

\author{
Arietta Spinou (i) ${ }^{1}$ and James D. Chalmers ${ }^{2}$ \\ Affiliations: 'Population Health Sciences, Life Sciences and Medicine, King's College London, London, UK. \\ ${ }^{2}$ School of Medicine, University of Dundee, Dundee, UK. \\ Correspondence: Arietta Spinou, Dept of Population Health Sciences, School of Population Health and \\ Environmental Sciences, 2nd floor, Addison House, Guy's Campus, King's College London, London, SE1 1UL, \\ UK. E-mail: arietta.spinoulakcl.ac.uk
}

@ERSpublications

A call for action for great awareness and research into airway clearance techniques and pulmonary rehabilitation in bronchiectasis http://bit.ly/2L8F4Va

Cite this article as: Spinou A, Chalmers JD. Respiratory physiotherapy in the bronchiectasis guidelines: is there a loud voice we are yet to hear? Eur Respir J 2019; 54: 1901610 [https://doi.org/10.1183/ 13993003.01610-2019].

Bronchiectasis is a chronic respiratory disease of airway dilatation, where patients typically suffer from respiratory infections, fatigue, sputum, cough, dyspnoea and poor quality of life $[1,2]$. This condition has received increased interest over the past years, with important developments in establishing national and international patient registries [3-5], randomised controlled trials of new treatments [6-8] and disease-specific health status questionnaires, such as the Bronchiectasis Health Questionnaire and the Quality of Life Questionnaire - Bronchiectasis $[9,10]$. A number of new treatment approaches have been proposed including long term antibiotic therapies and immune modulating drugs $[6,7,11,12]$.

Nevertheless the area of bronchiectasis care that has received the least attention during this period is the aspect of management that most healthcare professionals caring for bronchiectasis agree is the most important: airway clearance and exercise $[13,14]$. The evidence base for respiratory physiotherapy has not advanced at the same pace as other aspects of bronchiectasis care [15].

To assist clinical decision-making, bronchiectasis guidelines synthesise, evaluate the evidence and provide recommendations for clinical practice, and a number of clinical guidelines in bronchiectasis have been published since 2008 (table 1). Although usually similar, guidelines can present some variability in their recommendations even for the same topic [16-21]. This is particularly the case for physiotherapy, because of the limited evidence on which to base guideline recommendations. The purpose of this editorial is to discuss the current state of play worldwide with regard to airway clearance and pulmonary rehabilitation and suggest the need to prioritise research into these topics.

Current guidelines for the clinical management of bronchiectasis are available from national and international organisations: the European Respiratory Society (ERS 2017), British Thoracic Society (BTS 2019), Thoracic Society of Australia and New Zealand (TSANZ 2015), Spanish Society of Pulmonology and Thoracic Surgery (SEPAR 2018), Brazilian Thoracic Association (BTA 2019) and Saudi Thoracic Society (STS 2017) [16-21]. All guidelines refer to adults with bronchiectasis, whilst TSANZ and STS also include children $[20,21]$. Most documents are similar in that they are developed by multidisciplinary 
TABLE 1 Bronchiectasis clinical guidelines and their development methodology

\begin{tabular}{|c|c|c|c|c|}
\hline $\begin{array}{l}\text { Guideline } \\
\text { developers }\end{array}$ & Year & Databases of review & $\begin{array}{l}\text { Date of } \\
\quad \text { last search }\end{array}$ & $\begin{array}{l}\text { Level of evidence } \\
\text { appraisal system }\end{array}$ \\
\hline ERS & 2017 & $\begin{array}{c}\text { PubMed -MEDLINE-, Cochrane Central Register of } \\
\text { Controlled Trials (CENTRAL), Cochrane Library }\end{array}$ & Dec 2016 & GRADE \\
\hline BTS & 2010,2019 & $\begin{array}{l}\text { PubMed -MEDLINE-, EMBASE, Cochrane Library, } \\
\text { Database of Abstracts of Reviews of Effects (DARE), } \\
\text { Health Technology Assessment (HTA), NHS Economic } \\
\text { Evaluation Database (NHS EED) }\end{array}$ & June 2016 & SIGN \\
\hline TSANZ ${ }^{\#}$ & $2008,2010,2015$ & PubMed, Cochrane Central Library & Oct 2013 & Revised GRADE \\
\hline BTA $^{\text {T }}$ & 2019 & NR & NR & NR \\
\hline STS $^{\#}$ & 2017 & NR & NR & $\begin{array}{l}\text { Custom-made appraising }{ }^{+} \\
\text {(A, B, C , D ) }\end{array}$ \\
\hline
\end{tabular}

teams, used pre-defined PICO (patient, intervention, comparison, outcome) questions and used systematic literature searches to identify relevant evidence. To ensure integrity of the clinical guidelines, all members of the development panels disclosed conflict of interests, and in the ERS panel the conflicted members did not vote on recommendations that were of potential conflict. Moreover, some guidelines reported on the involvement of public or patient representatives [16, 18, 21].

Bronchiectasis guidelines discuss airway clearance techniques, mucoactive treatment and pulmonary rehabilitation to a variable extent, along with their corresponding evidence (table 2). The guidelines universally recommend that patients with bronchiectasis should be taught, and should practise, individualised airway clearance techniques and that this is best delivered by a respiratory physiotherapist (table 2). The grade of relevant recommendations ranges from weak (ERS, BTS) to strong (SEPAR, TSANZ) and the quality of relevant evidence is rated as low (SEPAR, ERS, BTS, STS) to moderate (BTS, TSANZ) [16-21]. Overall, airway clearance techniques show a short-term improvement in quality of life, cough-related measures and sputum volume expectoration compared to sham intervention and inactive control groups [22, 23]. A recent randomised controlled trial in 44 patients also demonstrated a reduction in exacerbations with twice daily airway clearance [24]. ERS, SEPAR, BTA and STS provide some examples of commonly practised airway clearance techniques. The BTS guideline recommends offering the active cycle of breathing techniques or oscillating positive expiratory pressure to patients, as well as considering gravity-assisted positioning when this is not contraindicated [18]. Still, little is known about the benefits of any specific technique over the others and data mainly come from small and short-term studies, often with a crossover design [22]. BTS additionally presents a list of clinical practice advice for airway clearance techniques and suggests management flowcharts during a clinically stable state and an acute exacerbation. This guideline discusses issues such as adherence, frequency of airway clearance techniques and review sessions, under "good practice points", derived from expert opinion [18].

Mucoactive treatments (expectorants, mucolytics, mucokinetics and mucoregulators) are also discussed in all bronchiectasis guidelines (table 2) [16-21]. These suggest offering long-term ( $\geqslant 3$ months) muco-active treatment in patients with difficulty expectorating sputum when standard airway clearance techniques are not adequate to control symptoms, rather than routinely. Most guidelines provide a strong recommendation against the use of recombinant human deoxyribonuclease (rhDNase) in bronchiectasis, as this is associated with higher exacerbation rate, more hospitalisations and a greater decline in forced expiratory volume in $1 \mathrm{~s}[25,26]$. BTS provides a weak recommendation to use humidification with sterile water or normal saline [18]. TSANZ advocates to consider mucoactive treatment, such as hypertonic saline and mannitol, in patients with frequent exacerbations rather than routinely, as longitudinal randomised controlled trials found that these provided little benefit over isotonic saline and placebo treatment, respectively $[27,28]$. There is therefore little clarity over when to use mucoactive treatments and how to define "difficulty expectorating sputum with standard airway clearance techniques". 
TABLE 2 Overview of respiratory physiotherapy recommendations in current clinical guidelines

Guidelines

Recommendation
Grade of

recommendation
Quality of

evidence

\section{Airway clearance techniques (ACT) ERS 2017}

BTS 2019

SEPAR 2018

TSANZ 2015

BTA 2019

STS 2017

Mucoactive treatment ERS 2017

BTS 2019

SEPAR 2018

TSANZ 2015

BTA 2019

STS 2017

\section{Pulmonary rehabilitation (PR) ERS 2017}

BTS 2019

SEPAR 2018

TSANZ 2015 BTA 2019
Teach ACT by respiratory physiotherapist

Perform ACT once or twice daily in chronic productive cough or difficulty to expectorate

Teach ACT to perform

Offer active cycle of breathing techniques or oscillatory positive expiratory pressure

Consider gravity assisted positioning where not contraindicated to enhance effectiveness of ACT

ACTs are safe

In stable bronchiectasis with productive cough (hypersecretion or frequent exacerbations)

Perform ACT

Get respiratory physiotherapist's advice

Individualise ACT

Teach and apply ACT to all patients with chronic production of secretions and/ or (CT scan) signs of mucus plugging

Lack of data about role of ACT in acute exacerbation, it may be used if no contraindications

Consider patient's preference and adherence to treatment

Teach patient or caregiver and encourage use of ACT and appropriate device

ACT is safe and may improve sputum expectoration, lung function and quality of life in stable bronchiectasis

Long-term mucoactive treatment in difficulty to expectorate sputum and poor quality of life, and where ACT cannot control symptoms

rhDNase is contraindicated

Consider use of humidification with sterile water or normal saline

Do not routinely use rhDNase

Insufficient evidence to recommend routine use of mucolytics

Hypertonic substances recommended in expectoration $>10 \mathrm{~mL}$ per day despite other treatment

Mucoactive agents are not routinely recommended

Consider trial in frequent exacerbations

rhDNase is contraindicated

Consider using hypertonic saline in persistent secretions despite other measures

Supervise first administration of hypertonic saline to assess adverse events, which can be prevented or minimised by prior administration of short-acting bronchodilator

Insufficient evidence to recommend routine use of mucolytics

DNase is contraindicated

Nebulised saline or mannitol may increase ease of sputum expectoration and decrease its viscosity

Be aware of airway hyperresponsiveness, as it occurs in $~ 12 \%$ of patients rhDNase is not recommended as potentially harmful

\section{Participate in PR}

Tailor to patient's symptoms, physical capability and disease characteristics Offer PR to those functionally limited by shortness of breath (mMRC $\geqslant 1$ )

Consider the use of inspiratory muscle training in conjunction to PR to enhance maintenance of the training effect

Participate in PR

Stable patients with mMRC dyspnoea scale $>1$

Participate in PR when exercise limitation

Refer to regular exercise and PR programme, if available

In clinically stable patients with chronic hypercapnic respiratory failure, non-invasive mechanical ventilation by BiPAP should be used as an adjuvant to cardiopulmonary rehabilitation

\begin{tabular}{|c|c|}
\hline Weak & Low \\
\hline Weak & Low \\
\hline D & 3 to $1-$ \\
\hline D & $1-$ \\
\hline D & $1-$ \\
\hline Strong & Low \\
\hline Strong & Low \\
\hline Strong & Moderat \\
\hline Strong & Moderat \\
\hline Strong & Moderat \\
\hline NR & NR \\
\hline NR & D \\
\hline NR & $D$ \\
\hline NR & D \\
\hline NR & C \\
\hline
\end{tabular}

Weak

Low

Strong

D

A

Strong

Strong

Weak

Weak

Strong

NR

NR

Moderate

3 to $1+$

$1+$

Low

Moderate

Moderate

Moderate

High

NR

NR

NR

NR

NR

NR

NR

NR

NR

D

Strong

Strong

B

High

High

1- to $1+$

$1-$ to $1+$

Strong

Strong

Strong

NR

Moderate

Moderate

Moderate

NR

NR 
TABLE 2 Continued

\begin{tabular}{llc} 
Guidelines & Recommendation & $\begin{array}{c}\text { Grade of } \\
\text { recommendation } \\
\text { evidence }\end{array}$ \\
\hline STS 2017 & $\begin{array}{l}\text { PR recommended as potential complementary option in moderate-to-severe } \\
\text { disease }\end{array}$ & NR
\end{tabular}

British Thoracic Society (BTS) grade of recommendation appraisal system referred to in the table: A: a systematic review of randomised controlled trials (RCTs) or a body of evidence consisting principally of studies rated as 1+ directly applicable to the target population and demonstrating overall consistency of results; B: a body of evidence including studies rated as 2++ directly applicable to the target population and demonstrating overall consistency of results or extrapolated evidence from studies rated as 1++ or 1+; D: evidence of level 3 or expert opinion or extrapolated evidence from studies rated as 2+. BTS level of evidence appraisal system referred to in the table: 1+: well conducted meta-analyses, systematic reviews of RCTs, or RCTs with low risk of bias; 1-: meta-analyses, systematic reviews of RCTs or RCTs with high risk of bias; 3: non-analytical studies, for example, case reports, case series. Saudi Thoracic Society (STS) system referred to in the table: A: RCTs with rich body of data or meta-analysis from RCTs; B: RCTs with a limited body of data or meta-analysis from non-RCTs; C: non-randomised trials and observational studies; D: panel consensus. ERS: European Respiratory Society; SEPAR: Spanish Thoracic Society; TSANZ: Thoracic Society of Australia and New Zealand; BTA: Brazilian Thoracic Association; STS: Saudi Thoracic Society; CT: computed tomography; NR: none reported; mMRC: modified Medical Research Council dyspnoea scale; BiPAP: bilevel positive airway pressure.

Regarding pulmonary rehabilitation, small ( $<85$ participants) and mainly short-term but well-conducted randomised controlled trials show clinically significant improvements in exercise tolerance and quality of life; incremental shuttle walk distance mean change $67 \mathrm{~m}$ (95\% CI 52 to $82 \mathrm{~m}$ ) and St George's Respiratory Questionnaire mean change -4.65 units $(-6.7$ to -2.6 units), respectively [26]. Nevertheless, a systematic review shows the evidence base for pulmonary rehabilitation consists of only four trials and 164 participants [29]. A recent study showed that pulmonary rehabilitation is equally effective in COPD and bronchiectasis [30]. There is a unanimously strong (ERS, SEPAR, TSANZ, BTS) recommendation for participating in pulmonary rehabilitation programmes when patients present impaired exercise capacity or report exercise limitation due to breathlessness (table 2). This recommendation was supported by moderate (TSANZ, SEPAR, STS) to high (ERS, BTS) quality of evidence [16-21]. When defined, exercise limitation due to breathlessness is indicated by a modified Medical Research Council dyspnoea scale score of $\geqslant 1$, which refers to the person that gets short of breath when hurrying on level ground or walking up a slight hill, or feels breathless even more easily than that. ERS, BTS and SEPAR consider the addition of inspiratory muscle training to support the maintenance of the exercise benefit, although there is no evidence of an additive benefit to exercise tolerance or other clinical outcomes [17, 18, 31]. Availability of pulmonary rehabilitation in many countries in Europe is limited due to lack of resources and the lack of evidence.

Guidelines support airway clearance and the use of mucoactive treatment based on low to moderate level of evidence, which indicates that further research could change our confidence in the estimated effect. On the other hand, pulmonary rehabilitation is supported by moderate to high quality of evidence, providing greater confidence on the effect of this treatment. Patients seem to accept and value airway clearance techniques and pulmonary rehabilitation but long-term compliance with them is unknown [32]. Moreover, airway clearance and pulmonary rehabilitation bronchiectasis recommendations largely rely on studies during a clinically stable stage. Thus, clinically, we are mainly called to extrapolate these results to disease exacerbations or assume that clinical outcomes will resemble those of other respiratory conditions; this could be misleading.

The evidence base for bronchiectasis therapy is evolving, but whereas several thousand patients have now been enrolled into trials of high cost therapies, such as inhaled antibiotics or inhaled mucoactive drugs, trials that have been largely unsuccessful, there have been no large trials of airway clearance and pulmonary rehabilitation [6, 8, 33]. A search of Clinicaltrials.gov and other trial databases identified no trials with >100 participants for these two interventions. The UK CLEAR trial of hypertonic saline and carbocisteine is an encouraging development that will recruit 380 patients (ISRCTN89040295). The absence of standardised, quality-assured airway clearance across centres participating in pharmacotherapy trials has been cited as one potential reason for their heterogeneous results [34, 35].

Preliminary data show that access to airway clearance techniques and pulmonary rehabilitation is highly variable in patients with bronchiectasis across Europe $[35,36]$. Bronchiectasis audits have also shown that implementation of the guidelines can be limited and even adherence to national standards of patient care is variable [37-40]. In the era of evidence-based medicine, evidence for what is considered usual care and a core part of bronchiectasis management is paramount for the implementation of the best available treatment. Once high-quality studies, evidence and strong clinical guidelines are available, our aim could focus on local implementation. Still, it seems a long way to that, and the question remains "how could we address these outstanding uncertainties as a community"? 
We issue a call to action, urging researchers, clinicians, funding bodies and the European Respiratory Society to prioritise airway clearance and pulmonary rehabilitation. Large randomised studies of these interventions are feasible, particularly with the availability of large registries and recently developed standardised endpoints [41].

Clinical guidelines since 2008 have underlined the need for high-quality evidence to support these therapies that bronchiectasis patients are recommended on a daily basis. Questions that remain unresolved include the role of airway clearance in stable disease and exacerbation, the optimum method of clearance, and its duration and frequency for improving long-term and clinically important outcomes in various disease severities. We need more data about the impact of airway clearance and pulmonary rehabilitation treatments in numerous areas, particularly exacerbations and hospitalisations; the role of physiotherapists in individualised action plans and home intravenous antibiotics; education in pulmonary rehabilitation; self-management; treatment compliance; and links between primary and secondary healthcare.

Airway clearance is largely individualised, and this remains a profound research challenge. It is acknowledged that large clinical trials including a comparator arm of no physiotherapy present ethical and logistical challenges, as airway clearance is a standard part of care and blinding is challenging. Innovative trial designs including cluster randomised approaches, active comparator studies or step-wedge implementation trials are alternatives which can overcome any barriers.

Great steps forward have been taken in changing bronchiectasis from an "orphan disease", but respiratory physiotherapy remains an orphan topic even in this "age of bronchiectasis". This has to change, for the benefit of all our patients.

Conflict of interest: A. Spinou has nothing to disclose. J.D. Chalmers reports grants and personal fees for consultancy, congress travel and/or lectures from GlaxoSmithKline, Grifols, Boehringer Ingelheim and Insmed, grants from AstraZeneca and Bayer Healthcare, personal fees for consultancy, congress travel and/or lectures from Aradigm, Pfizer and Napp, outside the submitted work.

\section{References}

1 Spinou A, Fragkos KC, Lee KK, et al. The validity of health-related quality of life questionnaires in bronchiectasis: a systematic review and meta-analysis. Thorax 2016; 71: 683-694.

2 Guan WJ, Huang Y, Chen CL, et al. Macrolides, mucoactive drugs and adherence for the management of bronchiectasis. Eur Respir J 2018; 51: 1701987.

3 Chalmers JD, Aliberti S, Polverino E, et al. The EMBARC European Bronchiectasis Registry: protocol for an international observational study. ERJ Open Res 2016; 2: 00081-2015.

4 Araujo D, Shteinberg M, Aliberti S, et al. The independent contribution of Pseudomonas aeruginosa infection to long-term clinical outcomes in bronchiectasis. Eur Respir J 2018; 51: 1701953.

5 Visser SK, Bye PTP, Fox GJ, et al. Australian adults with bronchiectasis: The first report from the Australian Bronchiectasis Registry. Respir Med 2019; 155: 97-103.

6 De Soyza A, Aksamit T, Bandel TJ, et al. RESPIRE 1: a phase III placebo-controlled randomised trial of ciprofloxacin dry powder for inhalation in non-cystic fibrosis bronchiectasis. Eur Respir J 2018; 51: 1702052.

7 Aksamit T, De Soyza A, Bandel TJ, et al. RESPIRE 2: a phase III placebo-controlled randomised trial of ciprofloxacin dry powder for inhalation in non-cystic fibrosis bronchiectasis. Eur Respir J 2018; 51: 1702053.

8 Haworth CS, Bilton D, Chalmers JD, et al. Inhaled liposomal ciprofloxacin in patients with non-cystic fibrosis bronchiectasis and chronic lung infection with Pseudomonas aeruginosa (ORBIT-3 and ORBIT-4): two phase 3, randomised controlled trials. Lancet Respir Med 2019; 7: 213-226.

9 Spinou A, Siegert RJ, Guan WJ, et al. The development and validation of the Bronchiectasis Health Questionnaire. Eur Respir J 2017; 49: 1601532.

10 Quittner AL, Marciel KK, Salathe MA, et al. A Preliminary Quality of Life Questionnaire-Bronchiectasis: a patient-reported outcome measure for bronchiectasis. Chest 2014; 146: 437-448.

11 De Soyza A, Pavord I, Elborn JS, et al. A randomised, placebo-controlled study of the CXCR2 antagonist AZD5069 in bronchiectasis. Eur Respir J 2015; 46: 1021-1032.

12 Altenburg J, de Graaff CS, Stienstra Y, et al. Effect of azithromycin maintenance treatment on infectious exacerbations among patients with non-cystic fibrosis bronchiectasis: the BAT randomized controlled trial. JAMA 2013; 309: 1251-1259.

13 Herrero-Cortina B, Vilaro J, Marti D, et al. Short-term effects of three slow expiratory airway clearance techniques in patients with bronchiectasis: a randomised crossover trial. Physiotherapy 2016; 102: 357-364.

14 Wong C, Sullivan C, Jayaram L. ELTGOL airway clearance in bronchiectasis: laying the bricks of evidence. Eur Respir J 2018; 51: 1702232.

15 Chalmers JD, Chotirmall SH. Bronchiectasis: new therapies and new perspectives. Lancet Respir Med 2018; 6: 715-726.

16 Polverino E, Goeminne PC, McDonnell MJ, et al. European Respiratory Society guidelines for the management of adult bronchiectasis. Eur Respir J 2017; 50: 1700629.

17 Martinez-Garcia MA, Maiz L, Olveira C, et al. Spanish Guidelines on treatment of bronchiectasis in adults. Arch Bronconeumol 2018; 54: 88-98.

18 Hill AT, Sullivan AL, Chalmers JD, et al. British Thoracic Society Guideline for bronchiectasis in adults. Thorax 2019; 74: Suppl. 1, 1-69. 
19 Pereira MC, Athanazio RA, Dalcin P, et al. Brazilian consensus on non-cystic fibrosis bronchiectasis. $J$ Bras Pneumol 2019; 45: e20190122.

20 Al-Jahdali H, Alshimemeri A, Mobeireek A, et al. The Saudi Thoracic Society guidelines for diagnosis and management of noncystic fibrosis bronchiectasis. Ann Thorac Med 2017; 12: 135-161.

21 Chang AB, Bell SC, Torzillo PJ, et al. Chronic suppurative lung disease and bronchiectasis in children and adults in Australia and New Zealand Thoracic Society of Australia and New Zealand guidelines. Med J Aust 2015; 202: 130.

22 Lee AL, Burge AT, Holland AE. Airway clearance techniques for bronchiectasis. Cochrane Database Syst Rev 2015; 11: CD008351.

23 Lee AL, Burge AT, Holland AE. Positive expiratory pressure therapy versus other airway clearance techniques for bronchiectasis. Cochrane Database Syst Rev 2017; 9: CD011699.

24 Munoz G, de Gracia J, Buxo M, et al. Long-term benefits of airway clearance in bronchiectasis: a randomised placebo-controlled trial. Eur Respir J 2018; 51: 1701926.

25 O'Donnell AE, Barker AF, Ilowite JS, et al. Treatment of idiopathic bronchiectasis with aerosolized recombinant human DNase I. rhDNase Study Group. Chest 1998; 113: 1329-1334.

26 Wilkinson M, Sugumar K, Milan SJ, et al. Mucolytics for bronchiectasis. Cochrane Database Syst Rev 2014; 5: CD001289.

27 Bilton D, Tino G, Barker AF, et al. Inhaled mannitol for non-cystic fibrosis bronchiectasis: a randomised, controlled trial. Thorax 2014; 69: 1073-1079.

28 Nicolson $\mathrm{CH}$, Stirling RG, Borg BM, et al. The long term effect of inhaled hypertonic saline 6\% in non-cystic fibrosis bronchiectasis. Respir Med 2012; 106: 661-667.

29 Lee AL, Hill CJ, McDonald CF, et al. Pulmonary rehabilitation in individuals with non-cystic fibrosis bronchiectasis: a systematic review. Arch Phys Med Rehabil 2017; 98: 774-782. e1.

30 Patel S, Cole AD, Nolan CM, et al. Pulmonary rehabilitation in bronchiectasis: a propensity-matched study. Eur Respir J 2019; 53: 1801264.

31 Chalmers JD, Crichton M, Goeminne PC, et al. The European Multicentre Bronchiectasis Audit and Research Collaboration (EMBARC): experiences from a successful ERS Clinical Research Collaboration. Breathe (Sheff) 2017; 13: 180-192.

32 McCullough AR, Tunney MM, Quittner AL, et al. Treatment adherence and health outcomes in patients with bronchiectasis. BMC Pulm Med 2014; 14: 107.

33 Polverino E, Dimakou K, Hurst J, et al. The overlap between bronchiectasis and chronic airway diseases: state of the art and future directions. Eur Respir J 2018; 52: 1800328.

34 Chotirmall SH, Chalmers JD. RESPIRE: breathing new life into bronchiectasis. Eur Respir J 2018; 51 : 1702444.

35 Flume PA, Chalmers JD, Olivier KN. Advances in bronchiectasis: endotyping, genetics, microbiome, and disease heterogeneity. Lancet 2018; 392: 880-890.

36 Walker P, Herrero B, Spinou A, et al. Variability in access and referral to pulmonary rehabilitation in European bronchiectasis patients enrolled in the EMBARC registry. Eur Respir J 2018; 52: Suppl. 62, OA5201

37 Herrero Cortina B, Aliberti S, Blasi F, et al. Chest physiotherapy in European patients with bronchiectasis: Data from the EMBARC registry. Eur Respir J 2017; 50: Suppl. 61, P A4071.

38 Aliberti S, Hill AT, Mantero M, et al. Quality standards for the management of bronchiectasis in Italy: a national audit. Eur Respir J 2016; 48: 244-248.

39 Hill AT, Routh C, Welham S. National BTS bronchiectasis audit 2012: is the quality standard being adhered to in adult secondary care? Thorax 2014; 69: 292-294.

40 Hill AT, Welham S, Reid K, et al. British Thoracic Society national bronchiectasis audit 2010 and 2011. Thorax 2012; 67: 928-930.

41 Hill AT, Haworth CS, Aliberti S, et al. Pulmonary exacerbation in adults with bronchiectasis: a consensus definition for clinical research. Eur Respir J 2017; 49: 1700051. 